\title{
The Importance of the Boundary Layer Parameterization in the Prediction of Low-Level Convergence Lines
}

\author{
Gerald L. Thomsen And Roger K. Smith \\ Meteorological Institute, University of Munich, Munich, Germany
}

(Manuscript received 1 March 2007, in final form 24 September 2007)

\begin{abstract}
The importance of the boundary layer parameterization in the numerical prediction of low-level convergence lines over northeastern Australia is investigated. High-resolution simulations of convergence lines observed in one event during the 2002 Gulf Lines Experiment are carried out using the fifth-generation Pennsylvania State University-National Center for Atmospheric Research Mesoscale Model (MM5). Calculations using five different parameterizations are compared with observations to determine the optimum scheme for capturing these lines. The schemes that give the best agreement with the observations are the three that include a representation of countergradient fluxes and a surface layer scheme based on MoninObukhov theory. One of these, the Medium-Range Forecast scheme, is slightly better than the other two, based on its ability to predict the surface pressure distribution. The findings are important for the design of mesoscale forecasting systems for the arid regions of Australia and elsewhere.
\end{abstract}

\section{Introduction}

In the Gulf of Carpentaria region of northeastern Australia, low-level convergence lines occur with great regularity at certain times of the year (Goler et al. 2006; Smith et al. 2006; Weinzierl et al. 2007). During the dry season, the lines are often marked by cloud lines such as the morning glory and the north Australian cloud line (NACL). "Morning glory" refers to a low-level rollcloud line or a series of such lines that arrive early in the morning in the southern gulf region. In this case the convergence line corresponds to a borelike disturbance. Such disturbances occur elsewhere in the world, often in association with cold fronts, or following the collision of gravity current-like flows such as sea breezes or thunderstorm outflows (Haase and Smith 1984; Smith 1986; Hoinka and Smith 1988). Their occurrence poses a threat to aircraft when taking off or landing, especially where they are not marked by cloud (Christie and Muirhead 1983). Other reports of cloud lines similar to the morning glory have been given by Clarke (1986),

Corresponding author address: Gerald L. Thomsen, Meteorological Institute, University of Munich, Theresienstr. 37, 80333 Munich, Germany.

E-mail: gerald@meteo.physik.uni-muenchen.de and a few noteworthy observations in other parts of the world include the fog waves over Berlin, Germany (Egger 1985), and the wave disturbance that occurred over the southern United Kingdom in 1914 (Billett 1914).

The NACL is a line of convective cloud that forms along the west coast of Cape York Peninsula early in the evening and moves westward across the Gulf of Carpentaria during the night and following day. In this case the convergence line corresponds to the leading edge of a gravity current (Thomsen and Smith 2006, hereafter TS06). During the dry season the clouds are mostly capped by the trade wind inversion at about 3 $\mathrm{km}$, but during the moist season they may develop as lines of thunderstorms that pose a significant forecasting problem in the region. A recent review of the various cloud lines is given by Reeder and Smith (1998).

The possibility of forecasting the convergence lines was examined by Jackson et al. (2002) using the mesoscale version of the Australian Bureau of Meteorology's Limited Area Prediction System (mesoLAPS). Their study was seen as a first step toward being able to forecast the formation and movement of lines of thunderstorms that the convergence lines sometimes trigger. Unfortunately, only satellite images were available for verification of the model forecasts, a fact that prompted the organization of the Gulf Lines Field Experiment 
(GLEX). Two phases of the experiment were carried out - the first was during September-October 2002 and the second was during November-December 2005. The overall aim of GLEX was to document the convergence lines and their accompanying clouds in as much detail as possible, and to provide a suitable database with which numerical predictions of the lines could be assessed. Details of the 2002 experiment are described by Goler et al. (2006), Smith et al. (2006), and Weinzierl et al. (2007).

High-resolution numerical simulations of two of the most interesting events from GLEX are described by TS06. For that study, which used the fifth-generation Pennsylvania State University-National Center for Atmospheric Research Mesoscale Model (MM5), it was necessary to determine the most appropriate boundary layer scheme for use from the many schemes available in the model. The choice of parameterization has a significant impact on the simulation because the processes leading to the generation of the lines, namely, the sea-breeze circulations around Cape York Peninsula, occur within the boundary layer. Although morning glory cloud lines are unique in that they occur relatively frequently in a particular geographical location, the generation of such disturbances is not confined to the Gulf of Carpentaria region. Moreover, the seabreeze circulations in this area are representative of those in a semiarid, coastal tropical climate. For these reasons, the results of our study are relevant to mesoscale simulations for many other regions.

Several recent studies have examined the capabilities of the boundary layer parameterizations available in MM5 in different situations. Tombrou et al. (2007) describe simulations using the Blackadar, GaynoSeaman, Medium-Range Forecast (MRF), and PleimXiu schemes. The MRF scheme was applied both in its original form and in a modified form that is more suitable for urban environments. Tombrou et al. examined a case for an urban area in a semihumid maritime subtropical climate and found that during nighttime stable conditions there is a need for improvements in all of the parameterizations. However, the overall performance of schemes in predicting the heights of the boundary layer during daytime was found to be close to that of the observations. Lee et al. (2005) focused on the nighttime stable conditions in a middle-latitude continental case, employing only the Blackadar scheme, the MRF scheme, and a version of the latter scheme with a modified nighttime module. They found that the MRF and Blackadar schemes overpredict the turbulent diffusion of heat during nighttime stable conditions, and thereby the height of the mixed layer. Berg and Zhong (2005) compared the Blackadar, Gayno-Seaman, and the MRF schemes in two cases-one being the same as that in Lee et al. and the other in a subtropical continental environment. They found that the mixed-layer depths were overpredicted by the MRF scheme and underpredicted by the Gayno-Seaman scheme, and were closest to those observed using the Blackadar scheme. The surface sensible heat flux was overpredicted by all schemes, an aspect that was attributed to inappropriate values for the soil moisture (SOILM). Deng and Stauffer (2006) focused their study mainly on the Gayno-Seaman scheme and a modified version thereof, with the vertical mixing of turbulent kinetic energy enhanced. A few calculations were also carried out using the MRF scheme. They selected a middlelatitude case in a maritime warm-temperate climate. They found that the MRF scheme overpredicted mixed-layer heights. The most comprehensive assessment of schemes was made by Zhang and Zheng (2004), who examined the Burk-Thompson, Eta, MRF, Gayno-Seaman, and Blackadar schemes. They describe model results for a subtropical continental case. Their methodology provided inspiration for our work, and therefore Zhang and Zheng's study is the most relevant to our paper. We discuss this paper in more detail in section 4. None of the cases described in any of these studies addresses climatic conditions similar to those in our case.

This paper compares simulations of one significant event during the GLEX experiment-that of 9 October 2002_ using the five different boundary layer schemes available in MM5. On this day, four disturbances were observed as seen in the satellite image shown in Fig. 1; these include morning glories moving from the northeast, the southeast, and the south, as well as an NACL. This event provides not only a suitable challenge for the model, but it is representative of other GLEX cases. The formation and evolution of the corresponding convergence lines in the five GLEX cases analyzed by Thomsen (2006), including the present one, showed many similarities. It was found also that the MRF scheme performed uniformly well in these cases, and the presumption here is that the other schemes have a similarly consistent performance.

The paper is organized as follows: Section 2 contains a brief description of the model configuration, including two land surface schemes that interface with particular boundary layer parameterizations (in the subsections we give an overview of the boundary layer schemes investigated), and follow in section 3 with a comparison of calculations using these schemes. In section 4 we attempt to isolate those aspects of the more 


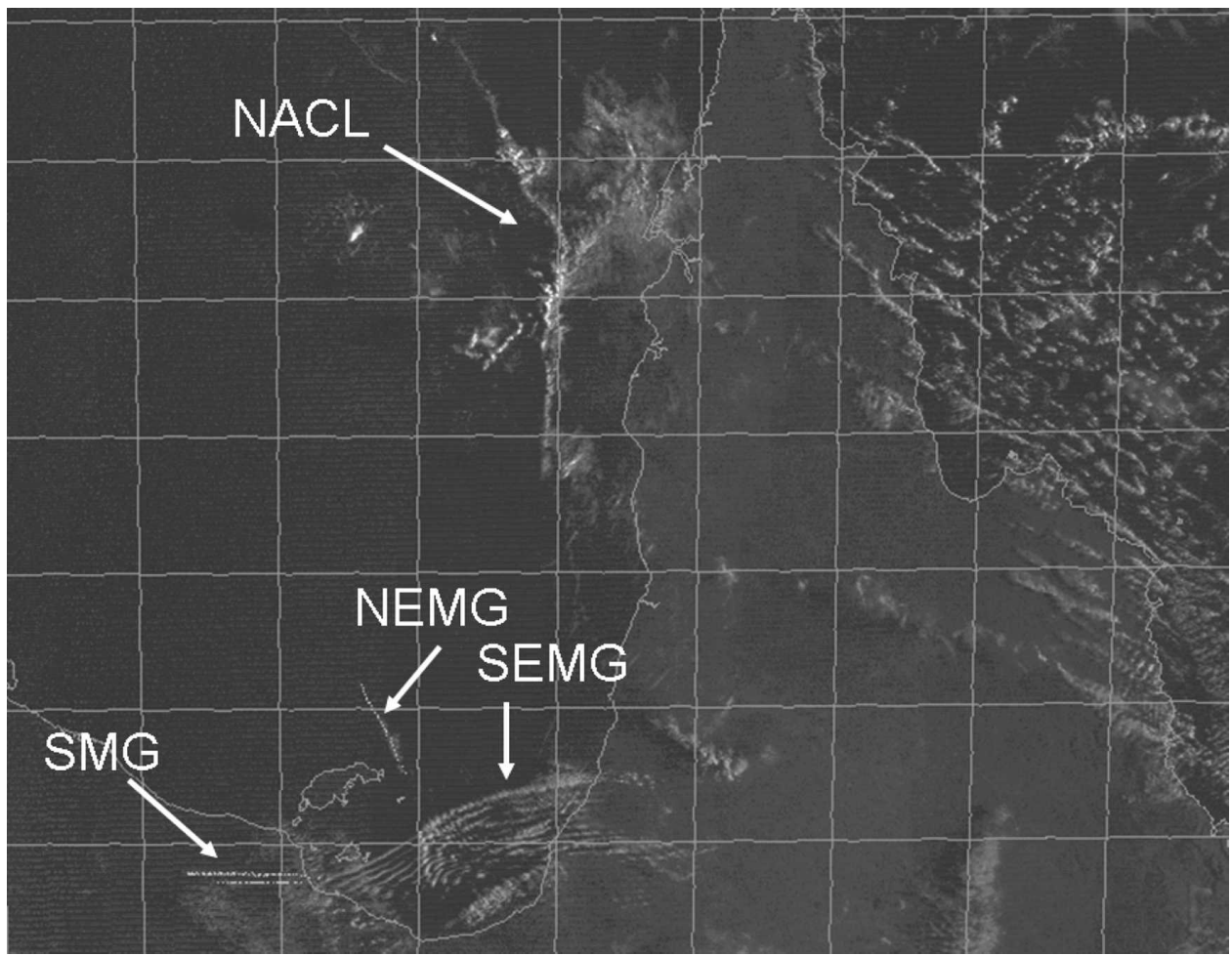

FIG. 1. Visible geostationary satellite image of the cloud lines at 0632 EST 9 Oct. Arrows indicate the northeasterly morning glory (NEMG), southeasterly morning glory (SEMG), southerly morning glory (SMG), and NACL.

superior schemes that are responsible for their skill, and in section 5 we present the conclusions.

\section{The model configuration}

Stull (1988) defines the planetary boundary layer as the part of the troposphere that is directly influenced by the presence of the earth's surface and responds to surface forcing with a time scale of about an hour or less. Over dry land surfaces in the tropics, the boundary layer can be as deep as $4 \mathrm{~km}$. In our model, there are 23 $\sigma$ levels providing relatively high resolution in the boundary layer. The 17 levels below $4 \mathrm{~km}$ are centered at heights of approximately 18, 54, 109, 182, 157, 332, 445, 599, 754, 994, 1322, 1662, 2016, 2383, 2767, 3168, and $3588 \mathrm{~m}$. Thus, the layer thicknesses in the lowest 4 $\mathrm{km}$ vary from $\sim 40$ to $\sim 400 \mathrm{~m}$ and increase with height.

The calculations are carried out on two horizontal domains shown in Fig. 2. The outer domain has $221 \times$ 221 grid points with a horizontal grid size of $9 \mathrm{~km}$ and the inner domain has $301 \times 301$ points with a grid size of $3 \mathrm{~km}$. The terrain land use and topography is taken from the U.S. Geological Survey dataset implemented in MM5 and has a 5' resolution in the outer domain and $2^{\prime}$ resolution in the inner domain. The time step is chosen to be $27 \mathrm{~s}$ for the outer domain and $9 \mathrm{~s}$ for the inner domain. The coupling of the two domains is performed every time step of the outer domain.

The Grell cumulus parameterization scheme (Grell 1993) is used in the outer domain, but no such scheme is used in the inner domain because the phenomena of interest involve predominantly dry processes. The Dudhia scheme is chosen as a parameterization for the microphysics (Dudhia 1989). The short- and longwave cloud and ground radiation schemestakes into account diurnal variations. Analysis data from the European Centre for Medium-Range Weather Forecasts (ECMWF) with a horizontal resolution of $0.25^{\circ}$ are used to provide initial and boundary conditions for the calculations.

The most energetic boundary layer eddies are tens of meters to $1-2 \mathrm{~km}$ in size in either the horizontal or vertical direction. The smaller eddies are not adequately resolved by our model resolution, which is typical for this type of model, so that a parameterization of the boundary layer is required. Five of the seven such parameterizations available in MM5 are investigated here. Those not considered are the Pleim-Xiu 
(a)

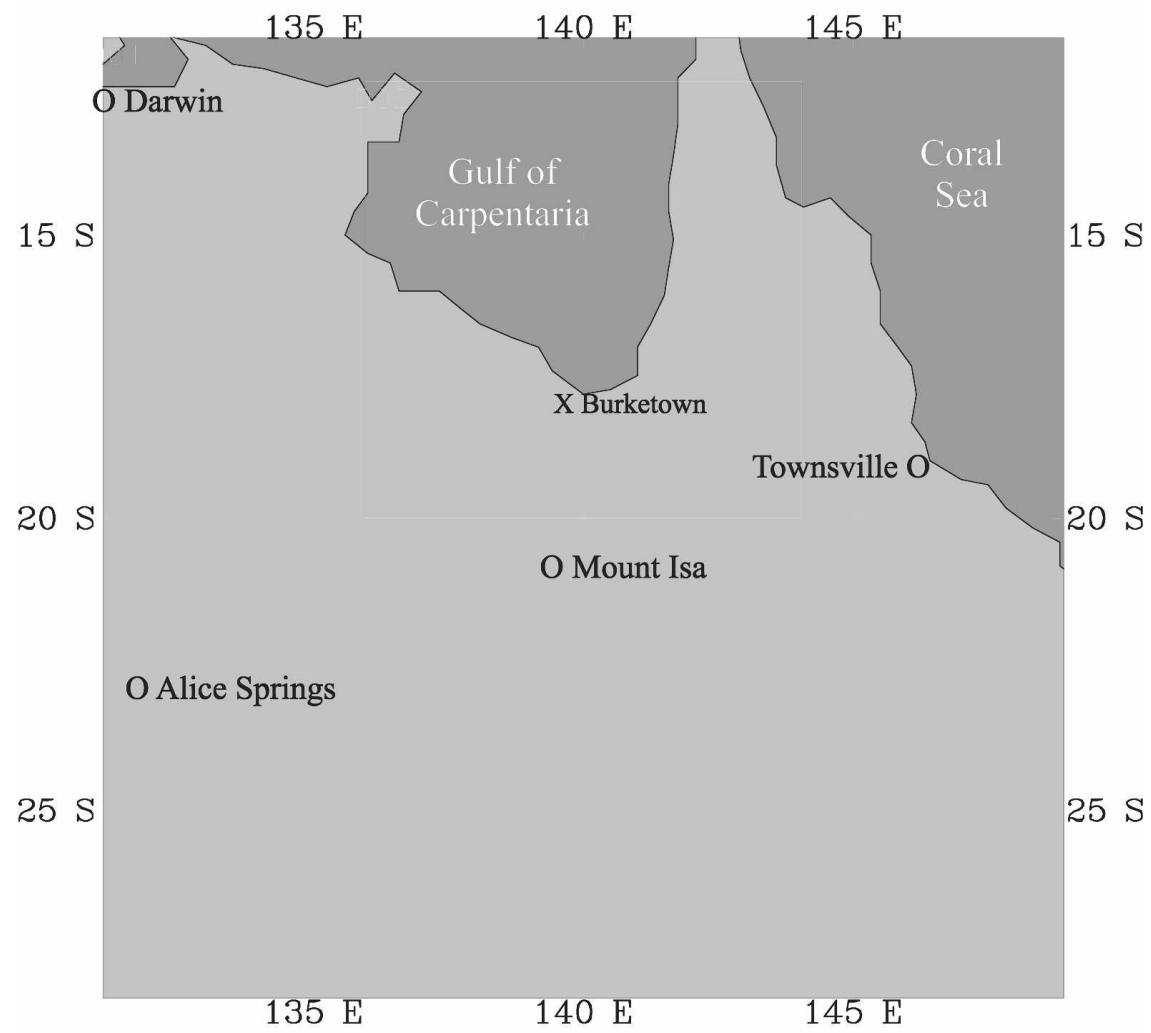

(b)

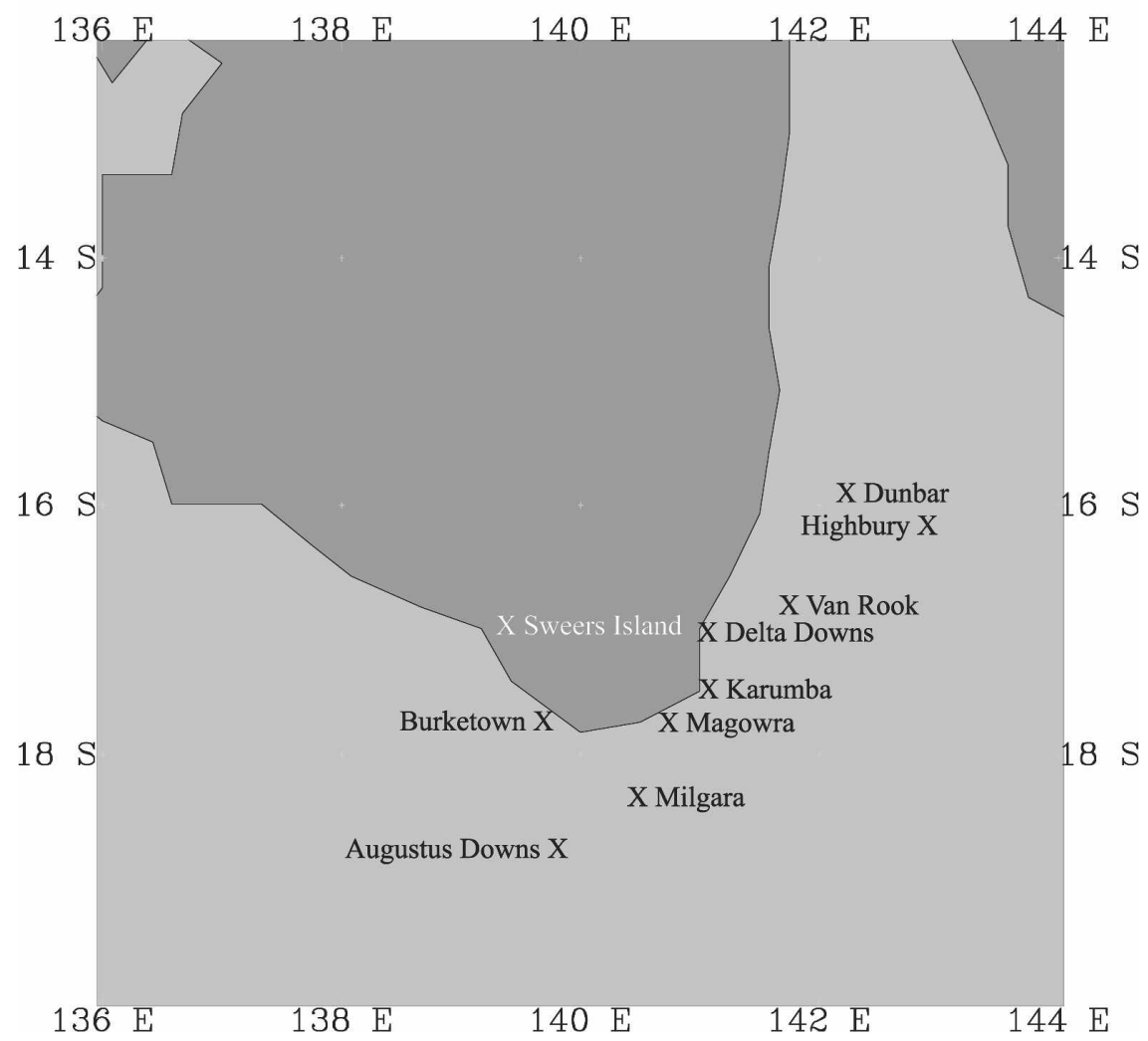


scheme, which is unavailable in a suitable version (a massive parallel version does not exist and the scheme is too demanding of memory for a single-processor run), and the bulk scheme, which led to numerical instability. The questions addressed below are as follows: how do the remaining schemes compare, and which have the most skill in forecasting low-level convergence lines over northeastern Australia?

In contrast to the simulations described in TS06, the ones here are carried out without a representation of moist vertical diffusion and without a soil moisture scheme. This is necessary because only a few boundary layer parameterizations in MM5 support both of these schemes. Moreover, we wish to isolate effects resulting solely from the choice of parameterization. Without the use of a soil moisture scheme, the soil moisture is held constant. The difference in runs using, or not using, this scheme can result only from changes of the soil moisture during the integration time. The average moisture availability (MAVA) in the inner domain is reduced only slightly (from about 0.45 to 0.44 ) in $24 \mathrm{~h}$ in the configuration used by TS06 (see Thomsen 2006, p. 104). The moist vertical diffusion option has its main impact in cloudy environments, in contrast to the case discussed here. The influence of this option has been assessed in Thomsen (2006, p. 77) and was found to be negligible. Thus, the present study is of direct relevance to that in TS06.

Each parameterization requires a land surface scheme of which four are available in MM5. These have various levels of sophistication, but not all of them can be interfaced with all the parameterizations. The two that interface with several parameterizations are the force-restore (Blackadar) scheme and the five-layer soil model. The former treats the soil as a single slab, which is assumed to span the depth over which there is a significant diurnal temperature variation (approximately $10-20 \mathrm{~cm}$ ). The slab temperature is based on the energy balance at the surface and the substrate temperature is fixed. The latter predicts the temperature in layers of 1-, 2-, 4-, 8-, and 16-cm depths using a vertical diffusion equation and assuming a fixed substrate temperature below the 16-cm-deep layer. The thermal inertia is derived in a similar way to the force-restore scheme except that the diurnal temperature variation is computed separately in the different layers, allowing for a more rapid response of the surface temperature.
The five-layer soil model is computationally more stable and allows for a larger time step than the forcerestore scheme. Both schemes use a "lookup" table for the surface characteristics like albedo, roughness, emissivity, and thermal inertia. These quantities are held constant during the model integration.

All simulations start at $1000 \mathrm{EST}^{1} 8$ October, and the integration time is $24 \mathrm{~h}$. We now review the main characteristics of the five boundary layer schemes investigated here.

\section{a. The MRF scheme}

The MRF scheme was developed initially for the National Centers for Environmental Prediction (NCEP) Medium-Range Forecast system by Hong and Pan (1996), and was implemented in MM5 by Dudhia and Hong, as stated in the corresponding piece of code. This scheme applies nonlocal $K$ mixing for the potential temperature and water vapor mixing ratio in the mixed layer, moist vertical diffusion in clouds, and local $K$ mixing above clouds. The nonlocal mixing is implemented following a nonlocal diffusion concept by Troen and Mahrt (1986). The term "nonlocal" as it appears in the literature is a little misleading. Here it refers to the flux of a particular quantity between adjacent layers calculated by applying a correction term for the local gradient. This correction term incorporates the contribution of the large-scale eddies to the total flux, thus allowing for countergradient fluxes. The eddy exchange coefficients $K$ are calculated from a prescribed profile function of boundary layer heights and scale parameters. The surface fluxes are calculated in the same way as in the Blackadar scheme. The four boundary layer stability states are determined using only the bulk Richardson number $\mathrm{Ri}_{b}$ : in the nighttime stable state $\left(\mathrm{Ri}_{b} \geq 0.2\right)$, all scaling parameters at the surface and all turbulent fluxes are set equal to zero; in the nocturnal damped mechanical turbulent state $(0<$ $\left.\mathrm{Ri}_{b}<0.2\right)$, the scaling parameters are determined by $\mathrm{Ri}_{b}$ and $L$ (the Monin-Obukhov length); in the nocturnal forced-convection state $\left(\mathrm{Ri}_{b}=0\right)$, the scaling parameters are determined by the local Richardson number Ri only (local $K$ theory); and when $\mathrm{Ri}_{b}<0$, the

\footnotetext{
${ }^{1}$ All dates and times are given in eastern Australian time (EST), which is $10 \mathrm{~h}$ ahead of coordinated universal time (UTC).
}

$\leftarrow$

Fig. 2. Map of northeastern Australia showing (a) the outer domain of the model with places mentioned in the text, and (b) the inner domain with the locations of AWSs in the southern gulf region marked by crosses. 
daytime module is used and the countergradient terms take effect.

\section{b. The Blackadar scheme}

The Blackadar scheme (Blackadar 1976) was first introduced as a representation of the nocturnal boundary layer. The version used here was extended by Blackadar $(1978,1979)$ and Zhang and Anthes (1982) to account also for the daytime boundary layer. The present scheme has two modules: one for the daytime convective state and one for the nighttime stable state. The module that is invoked depends on the vertical temperature gradient in the lowest model layer and on the magnitude of $\left|z_{h} / L\right|$, where $z_{h}$ is the height of the mixed layer and $L$ is the Monin-Obukhov length. The vertical temperature gradient in the lowest model layer is characterized by $\mathrm{Ri}_{b}$. In the nocturnal module, the atmosphere is assumed to be stably stratified, or at most slightly unstable, and a first-order closure scheme is used. To account for the largest gradients, which generally occur in the lowest layer, a surface layer of $10-\mathrm{m}$ depth is used, based on Monin-Obukhov similarity theory. The module for the nocturnal boundary layer is subdivided into three stability states, which are the same as those described in section $2 \mathrm{a}$. The nighttime stable state is assumed when $\mathrm{Ri}_{b} \geq 0.2$, the damped mechanical turbulent state when $0<\mathrm{Ri}_{b}<0.2$, and the forced convection state when $\mathrm{Ri}_{b} \leq 0$ and $\left|z_{h} / L\right| \leq 1.5$. The daytime module allows for free convection and is active when $\mathrm{Ri}_{b} \leq 0$ and $\left|z_{h} / L\right| \geq 1$.5. Discrete matrix forms of nonlocal theory are then used to parameterize convective circulations. In nonlocal theory, the vertical transfer of momentum, heat, and moisture is not determined by the local mean gradient, but by the thermal structure of the whole mixed layer. The Blackadar scheme is the only one of those studied that applies transilient mixing in any of the stability states, which allows mixing between nonadjacent vertical layers. It is also the only boundary layer parameterization in MM5 that works with both the force-restore and the fivelayer surface schemes.

\section{c. The Mellor-Yamada-based schemes}

All three Mellor-Yamada-based schemes described here use a one-and-a-half-order closure, which refers to level 2.5 in the Mellor-Yamada hierarchy (Mellor and Yamada 1974). A comprehensive summary of the different closures is given by Stull $(1988,197-250)$. In a one-and-a-half-order closure the eddy exchange coefficient of an adiabatically conserved quantity is related to the predicted turbulent kinetic energy (TKE). This kind of scheme is often referred to as a "TKE scheme."

\section{1) The Gayno-Seaman scheme}

The Gayno-Seaman scheme is a level-2.5 MellorYamada-based scheme. To represent cloud water in a consistent way, the model uses liquid water potential temperature $\theta_{L}$ and total water mixing ratio $q_{T}$, which are both conserved thermodynamic variables in nonprecipitating clouds (Betts 1973). The turbulent vertical transport of $\theta_{L}$ is parameterized using a countergradient heat flux term, based on the sensible heat flux, boundary layer height, and the convective vertical velocity scale (Therry and Lacarrere 1983). The GaynoSeaman scheme is the only one in MM5 for which TKE is treated as a prognostic quantity and for which TKE is advected. The scheme interfaces with the five-layer soil model. The surface fluxes for the Gayno-Seaman scheme are based on the same Monin-Obukhov similarity parameterization used with the Blackadar scheme, and the stability states are determined using the same criteria (Shafran et al. 2000). The boundary layer heights are calculated for all stability states except for the stable state.

\section{2) The EtA scheme}

The Eta scheme was originally implemented by Janjić (1990, 1994) in the NCEP Eta (step mountain) Model. It is based on the level-2.5 Mellor-Yamada scheme above the surface layer. The treatment of the surface layer is different over land and sea. In the original version, a Mellor-Yamada level-2 scheme was used for the surface layer, but in the MM5 version, the surface fluxes are calculated according to similarity theory. As in the Burk-Thompson scheme, horizontal advection, diffusion, and vertical advection of TKE are ignored. The Eta scheme interfaces with the MM5 fivelayer soil model, a requirement arising from the long time step of the Eta scheme.

\section{3) The Burk-Thompson scheme}

The Burk-Thompson scheme was originally designed for the marine boundary layer (Burk and Thompson 1982) and incorporated both level 2.5 and 3.0 schemes. The early versions of the scheme, which were implemented in the U.S. Navy's Navy Operational Regional Atmospheric Prediction System (NORAPS), apply a higher vertical resolution than that in the model and include a countergradient flux term for temperature. These two features are not implemented in MM5 and only the level-2.5 version is available. The BurkThompson scheme has its own force-restore ground temperature scheme and does not interface with any other MM5 soil models or land use schemes. The Louis (1979) scheme is used to parameterize the surface layer 
and applies an empirical fit to the Businger profile functions. Neither horizontal advection, diffusion, nor vertical advection of TKE are included in this scheme.

\section{Results}

A prerequisite for the TS06 study was determination of the optimum boundary layer scheme for forecasting low-level convergence lines that develop over northeastern Australia. It was necessary to compare the predictions using the different schemes and to determine the sensitivity of the forecasts to the choice of scheme. We report on this below.

A qualitative assessment of the model skill is provided by comparing the location and orientation of the convergence lines in the model with the cloud lines observed in satellite imagery. Surface pressure is an appropriate quantity to assess the quality of the model results because surface observations during GLEX showed that the passage of the cloud lines coincides with marked change in the wind speed and direction and in the surface pressure (TS06, see their Fig. 4). On the other hand, there is no coherent signature in either temperature or dewpoint. Figure 3 shows the sea level pressure at Karumba during in the simulation. The peak at about 0600 EST corresponds to either the northeasterly or southerly morning glory or both, because both lines arrived at Karumba at nearly the same time.

A quantitative measure of the model skill is obtained by calculating a correlation coefficient between the model-predicted and observed surface pressure. In the tropics, however, surface pressure has large diurnal and semidiurnal signals on account of the atmospheric tide and the heating and cooling of the atmosphere. Therefore, a sharper measure of the correlation is obtained by filtering these signals from the observational data and the model output before correlating these time series. The filtering was accomplished by Fourier analyzing the time series and then subtracting the lowest two frequencies. The correlation coefficient is defined by

$$
r_{\mathrm{MSLP}}=\frac{\sum\left(p_{\mathrm{obs}}-\overline{p_{\mathrm{obs}}}\right)\left(p_{\mathrm{mod}}-\overline{p_{\mathrm{mod}}}\right)}{\sqrt{\sum\left(p_{\mathrm{obs}}-\overline{p_{\mathrm{obs}}}\right)^{2} \sum\left(p_{\mathrm{mod}}-\overline{p_{\mathrm{mod}}}\right)^{2}}},
$$

where the average is taken over the stations shown in Fig. 2b. The index "obs" refers to a measured value, "mod" refers to the model value, and variables with an overbar are averaged over the 24-h forecast period. There were no automatic weather stations (AWSs) in the gulf, except for the two situated on Sweers Island and Mornington Island in the southern gulf, but morning glory cloud lines are more or less straight and occur
Karumba 081002

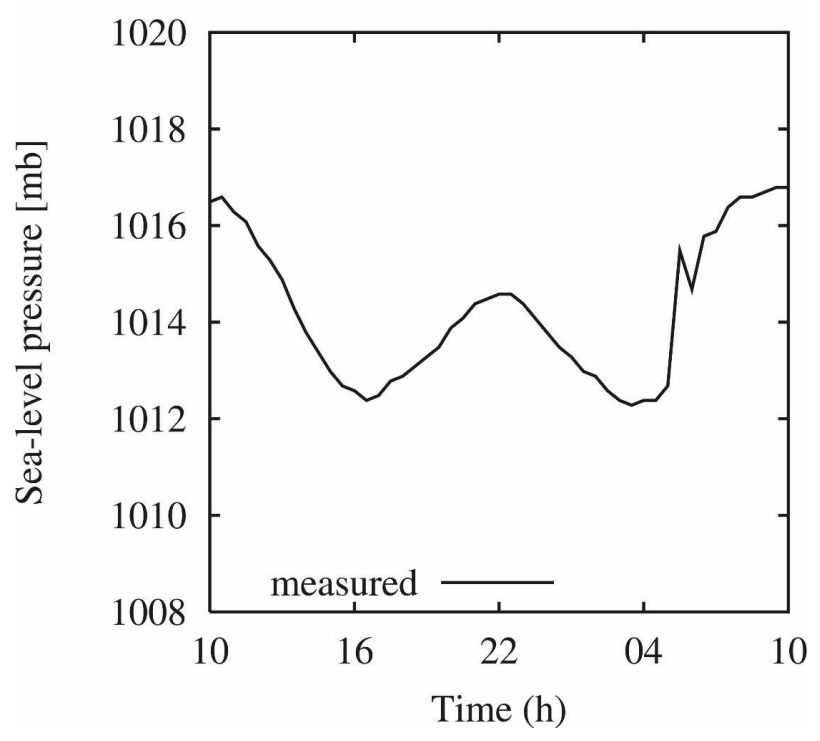

FIG. 3. Sea level pressure at Karumba during the simulation time.

over land as well as over the sea. Therefore, the data obtained should be adequate to capture the essence of the cloud lines. The Australian Bureau of Meteorology does maintain a few additional AWSs in the Gulf of Carpentaria region, but the data at the time of GLEX were available only every $3 \mathrm{~h}$, which is too infrequent for our study.

Table 1 lists properties of the boundary layer schemes as well as the mean sea level pressure (MSLP) correlation coefficients for the experiments with the different boundary layer parameterizations described in the previous section. The results can be subdivided into three groups in terms of the magnitude of this coefficient. The Blackadar, MRF, and Gayno-Seaman schemes give the highest correlation, the Eta and BurkThompson schemes give a lower correlation, and explicit modeling of the boundary layer gives the worst correlation. The crucial differences between the best schemes and those with a more modest skill are discussed below.

Figure 4 shows the low-level divergence field and wind vectors at 0600 EST 9 October for calculations with each of the five parameterizations and for one calculation in which no such scheme was used.

The panels are arranged in descending order of the MSLP correlation coefficient. The convergence line corresponding to the NACL is labeled "A" in each panel and those corresponding to the northeasterly morning glory and the southerly morning glory are labeled "B" and "C," respectively. The position of the cold front to the south of the gulf is labeled "D." 
TABLE 1. Experiments with the different PBL schemes, important groups of their properties, and mean sea level correlation coefficients. Here, $\theta$ refers to the potential temperature, $q$ to the water vapor mixing ratio, $u$ to the zonal wind, $v$ to the meridional wind, and $\theta_{L}$ to the liquid water potential temperature.

\begin{tabular}{|c|c|c|c|c|c|c|}
\hline Expt & PBL scheme & Closure (order) & Surface fluxes & $\begin{array}{l}\text { Countergradient } \\
\text { fluxes* }\end{array}$ & Land surface scheme & $r_{\text {MSLP }}$ \\
\hline 1 & MRF & Profile (1) & Monin-Obukhov similarity & $\theta, q$ & Five layer & 0.925 \\
\hline 2 & Blackadar & Local Ri $(1)^{* *}$ & Monin-Obukhov similarity & $\theta, q, u, v$ & Force-restore & 0.924 \\
\hline 3 & & & & & Five layer & 0.924 \\
\hline 4 & Gayno-Seaman & TKE (1.5) & Monin-Obukhov similarity & $\theta_{L}$ & Force-restore & 0.908 \\
\hline 5 & Eta & TKE (1.5) & Similarity theory & & Five layer & 0.876 \\
\hline 6 & Burk-Thompson & TKE (1.5) & Louis scheme & & Force-restore & 0.863 \\
\hline 7 & Explicit & & & & Force-restore & 0.618 \\
\hline
\end{tabular}

* Daytime convective stability state only.

** Nighttime stable states only.

Two tests of the Blackadar scheme were made, one using the force-restore land surface scheme and the other using the five-layer scheme. The value of $r_{\text {MSLP }}$ (0.924) turned out to be the same for the two calculations, and the convergence patterns are so similar that we show only the results for the force-restore scheme. One deduction from the similarity of the convergence patterns is that the surface fluxes with both surface schemes must be very similar.

The first available visible satellite image was that at 0632 EST, 32 min after the time shown in the plots. In this image, the NACL is located along the western side of the $141^{\circ}$ meridian. Based on backward extrapolation using the images at 0632 and $0825 \mathrm{EST}$, its location at 0600 EST would have been approximately $12 \mathrm{~km}$ east of this meridian. All model runs captured a convergence line too far west of the cloud location, indicating that the line travels too fast in all simulations. The line was captured closest to the estimated position with the Eta scheme $(12 \mathrm{~km})$, and the farthest was with the Burk-Thompson scheme $(57 \mathrm{~km})$ and without a PBL scheme $(64 \mathrm{~km})$. In the runs with the MRF, GaynoSeaman, and Blackadar schemes, the line was $38 \mathrm{~km}$ too far west. The error in assessing the position of the lines is estimated to be $\pm 10 \mathrm{~km}$. There are large differences also in the structure of the convergence line between the runs. The line is most coherent when using the MRF scheme and, rather surprisingly, in the run without any scheme, but it is rather fragmented at its northern end in the other four simulations.

In the satellite image at $0632 \mathrm{EST}$, the northeasterly morning glory passes through $17^{\circ} \mathrm{S}, 140^{\circ} \mathrm{E}$, and the backward extrapolation with the later image leads to an estimated position at 0600 EST that is $13.5 \mathrm{~km}$ to the northeast. The corresponding convergence line in the model is captured most accurately about $12 \mathrm{~km}$ from the observed position by the MRF scheme. In comparison, the location in the simulations using the Gayno-
Seaman, Blackadar, Burk-Thompson, and Eta schemes is about $25,31,70$, and $84 \mathrm{~km}$ too far to the northeast, respectively. The line is displaced only about $38 \mathrm{~km}$ to the northeast using no scheme, but its Y-shaped northwestern end predicted using no scheme was not observed. The error in determining the position of the lines in the model is estimated to be at most $\pm 10 \mathrm{~km}$. The length of the convergence lines cannot be compared with those in satellite imagery because the length in the divergence plots depends on the contour interval chosen.

The most striking difference between the model results is the convergence line corresponding to the southerly morning glory. The three simulations with the largest values of $r_{\text {MSLP }}$ produced a well-pronounced line. Using the Eta and Burk-Thompson schemes, the convergence line is barely perceptible. In Fig. $4 \mathrm{~d}$ it is only just detectable by a small bulge in the northeasterly morning glory convergence line. Plots of the field before and after, also with different contour intervals, show that the bulge definitely corresponds to the southerly morning glory. However, at this time, the position closely coincides with the position of the NACL as well, and the bulge also could be related to the NACL. Its position in Fig. $4 \mathrm{e}$ is revealed by a small break in the convergence line corresponding to the northeasterly morning glory and some smaller regions of increased convergence. The convergence line corresponding to the southerly morning glory is located about $200 \mathrm{~km}$ too far south in the run without a scheme. No scheme was able to distinguish between the southerly and southeasterly morning glories that are seen in Fig. 1.

The cold front to the south of the gulf is not marked everywhere by strong convergence, but it is marked by a strong change in wind speed and direction. The western side of the convergence line corresponding to the southerly morning glory is connected to the cold front using the MRF and especially the Gayno-Seaman 
(a)

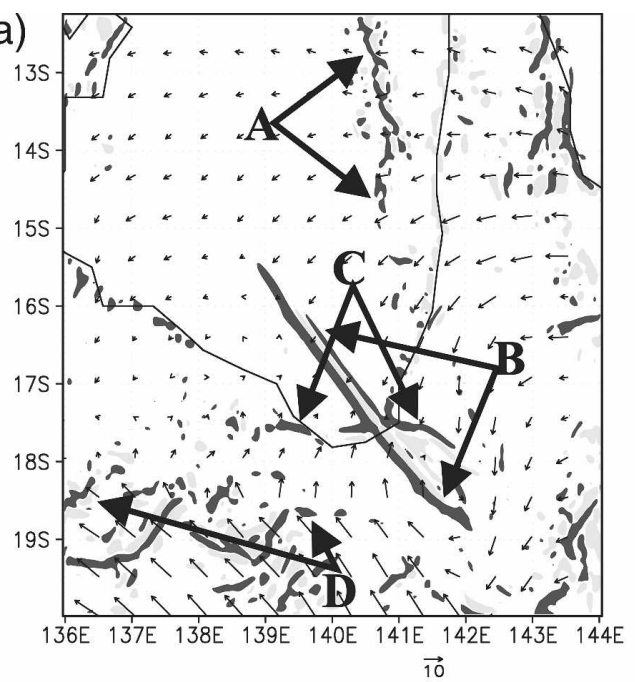

(c)

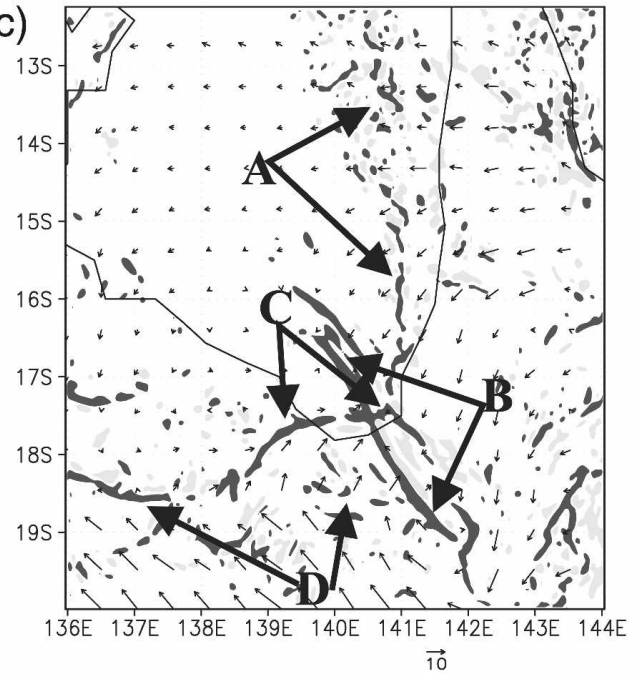

(e)

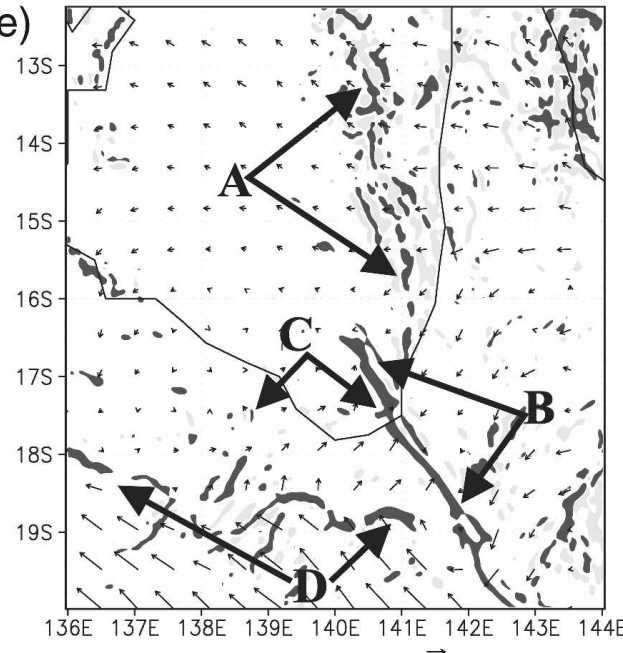

(b)

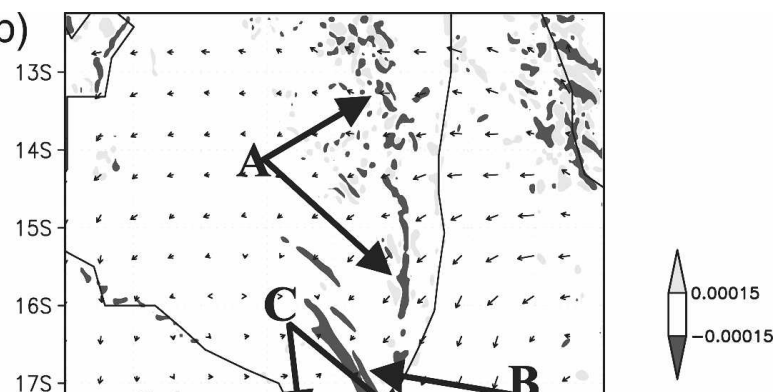

(d)
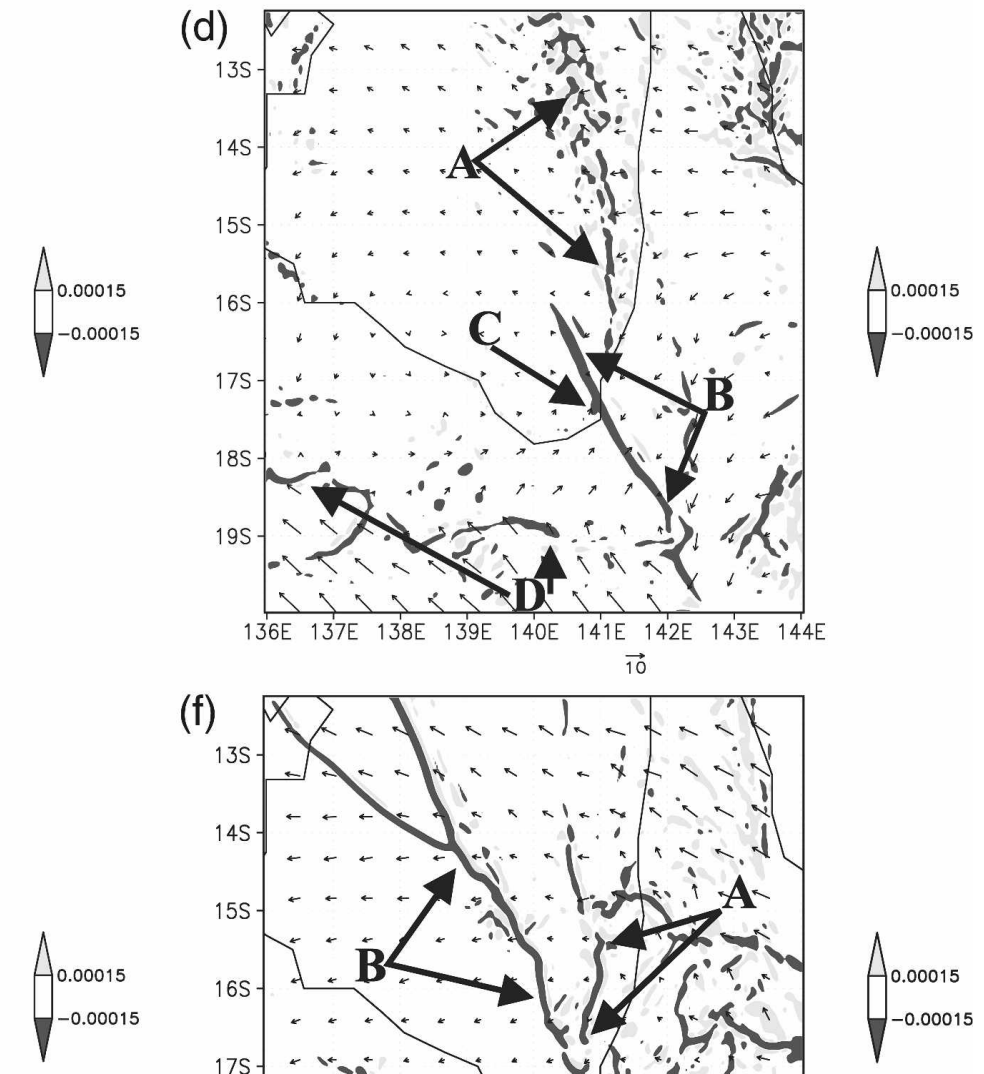

(f)

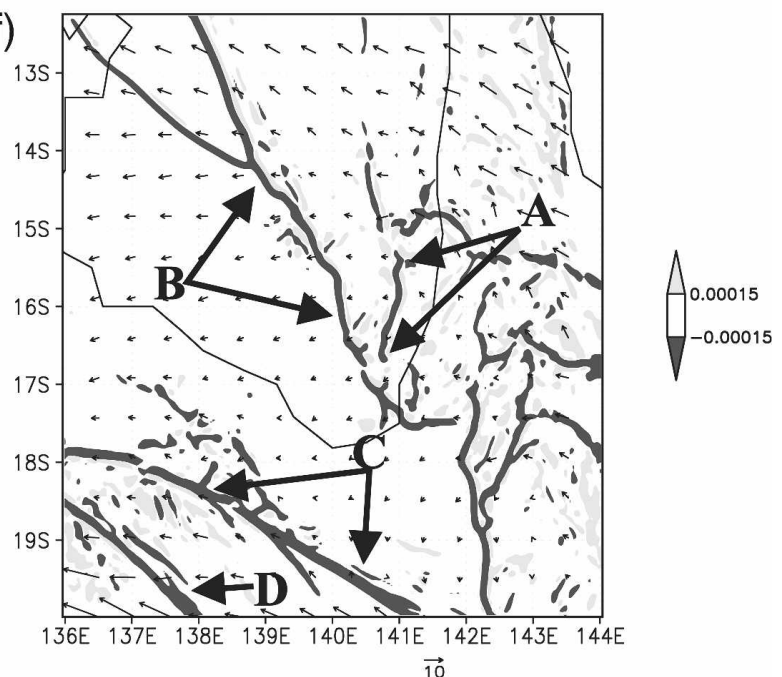

FIG. 4. Low-level wind vectors (scale $10 \mathrm{~m} \mathrm{~s}^{-1}$, shown below panels) and divergence (shaded, $\mathrm{s}^{-1}$ ) at $\sigma=0.955$ at 0600 EST 9 Oct. The capital letters stand for convergence lines corresponding to an NACL (A), a northeasterly morning glory (B), a southerly morning glory (C), and a cold front (D). 
scheme, but this southwest-to-northeast-oriented part of the line is located too far west to be interpreted as a southeasterly morning glory.

\section{Discussion}

The three boundary layer schemes giving the highest values of $r_{\mathrm{MSLP}}$ are the Blackadar, Gayno-Seaman, and MRF schemes. The value of $r_{\text {MSLP }}$ varies only slightly between these three schemes and is greater than 0.90 . These schemes have the following two features in common:

1) the treatment of the surface layer and

2) the representation of countergradient fluxes in the daytime convective module.

In the Blackadar scheme, the countergradient fluxes of the water vapor mixing ratio, potential temperature, and horizontal wind speeds are represented by the transilient scheme. In the Gayno-Seaman scheme, the turbulent fluxes of the liquid water potential temperature are calculated using a countergradient heat flux term. In the MRF scheme, the turbulence diffusion equations for potential temperature and water vapor mixing ratio apply a countergradient correction term. The common feature of the schemes is that they all allow for countergradient fluxes of a form of the potential temperature, which suggests that the countergradient flux of this quantity is more important than the flux of moisture and momentum.

The Eta and Burk-Thompson schemes differ from the above three schemes in their treatment of the surface layer and the fact that they do not have any representation of countergradient fluxes. The value of $r_{\text {MSLP }}$ for these schemes is 0.876 and 0.863 , respectively. The simulation without a boundary layer parameterization rates poorly in comparison with all schemes, giving a value of $r_{\text {MSLP }}=0.618$.

The difference between the values of $r_{\text {MSLP }}$ for the MRF scheme and for the Eta and Burk-Thompson schemes is about 0.049 and 0.062 , respectively. We try now to quantify the relative effects that the treatment of the surface layer and the representation of countergradient fluxes have on the skill of the MRF scheme as measured by the value of $r_{\text {MSLP. }}$. Both the countergradient mixing and surface layer scheme have the transport of heat from the surface or lower levels to higher levels in common, and thus the sea-breeze circulation is affected. The correct timing of the sea breezes, in turn, has a paramount impact on the formation and orientation of the low-level convergence lines corresponding with the morning glories and the NACL. Morning glories and sea-breeze fronts have a strong surface pres- sure signature, and it seems appropriate, therefore, to focus on $r_{\text {MSLP. }}$

First we assess the importance of the countergradient fluxes by suppressing their effect in the MRF scheme. To do this we carried out two additional experiments with this scheme-one with the nocturnal stable state only and the other with the free convective state only. With the nocturnal state switched off, the results were essentially the same, indicating that it is the inclusion of the free convective state that is of paramount importance. When only the nocturnal state is invoked, the convergence line corresponding to the northeasterly morning glory at 0600 EST 9 October is located about $100 \mathrm{~km}$ too far northeastward, but with little difference in intensity. The convergence line corresponding to the southerly morning glory arrives at the gulf coast at about the same time as observed, while its intensity is weaker in this calculation than with the unmodified MRF configuration. Switching the countergradient fluxes off reduces the value of $r_{\mathrm{MSLP}}$ by 0.027 . Thus, the countergradient fluxes account for approximately $54 \%$ and $43 \%$ of the difference between $r_{\mathrm{MSLP}}$ for the MRF and $r_{\mathrm{MSLP}}$ for the Eta and Burk-Thompson schemes, respectively.

We turn now to the surface layer representation, which is important because the surface sensible heat fluxes have a major influence on the sea-breeze circulations. In turn, the intensity of these circulations affects the intensity and propagation speed of the morning glory convergence lines. This dependence is shown in a sensitivity study on the effects of soil moisture carried out by Thomsen (2006). In general, the Bowen ratio becomes smaller as the surface becomes moister, but it is the sensible heat flux that directly drives the sea-breeze circulation. Thus, the MSLP correlation coefficient declines as the soil moisture increases.

The model continues to generate the convergence lines as the surface moisture is increased, at least up to the largest moisture value examined (MAVA $=0.47$ ), but the timing of the lines is progressively delayed compared with the observations, consistent with the weaker sea-breeze circulations.

Zhang and Zheng (2004) performed a sensitivity study of MM5 to five different boundary layer parameterizations, comparing the surface temperature $T_{\mathrm{SFC}}$ among these schemes, where $T_{\mathrm{SFC}}=0.45 T_{G}+0.55 T_{A}$, and $T_{G}$ and $T_{A}$ are the ground temperature and the temperature in the lowest model layer, respectively. They found that the maximum surface temperature was underestimated by $1^{\circ}-2^{\circ} \mathrm{C}$ when using the BurkThompson and Eta schemes, and they attributed this underestimate to the surface layer scheme, which differs from those in the parameterizations that showed 
TABLE 2. Experiments with the different boundary layer schemes, average surface temperature $\overline{\left(T_{\mathrm{SFC}}\right)}$ at 1600 EST 8 Oct, and MSLP correlation coefficient ( $\left.r_{\mathrm{MSLP}}\right)$, which the MRF scheme would produce in an experiment in which the surface layer temperature was adjusted to this value by the soil moisture.

\begin{tabular}{clcc}
\hline \hline Expt & PBL scheme & $\overline{T_{\mathrm{SFC}}}\left({ }^{\circ} \mathrm{C}\right)$ & $\begin{array}{r}r_{\mathrm{MSLP}}(\mathrm{MRF}) \\
\text { with } \overline{T_{\mathrm{SFC}}}\end{array}$ \\
\hline 1 & MRF & 31.72 & 0.923 \\
2 & Blackadar & 31.18 & 0.912 \\
3 & & 31.16 & 0.912 \\
4 & Gayno-Seaman & 32.08 & 0.929 \\
5 & Eta & 30.98 & 0.908 \\
6 & Burk-Thompson & 30.31 & 0.891 \\
7 & Explicit & 24.30 & 0.654 \\
\hline
\end{tabular}

no such underestimate. In the present calculations, the spatial average of the surface temperature at 1600 EST 8 October is about $1 \mathrm{~K}$ lower in the Eta and BurkThompson schemes than in the other three (see third column in Table 2), which is in line with Zhang and Zheng's findings.

We attempt to quantify the importance of the surface layer representation by reducing the surface temperature in the MRF scheme to the same as that in the Eta and Burk-Thompson schemes and examining the change in the value of $r_{\text {MSLP. }}$ One way to reduce the surface temperature is to increase the soil moisture. We apply the results of Thomsen's (2006) study of the sensitivity to soil moisture to quantify the effects of the reduced surface temperature. As in the previous section we removed the diurnal and semidiurnal components of the surface pressure before calculating the

(a)

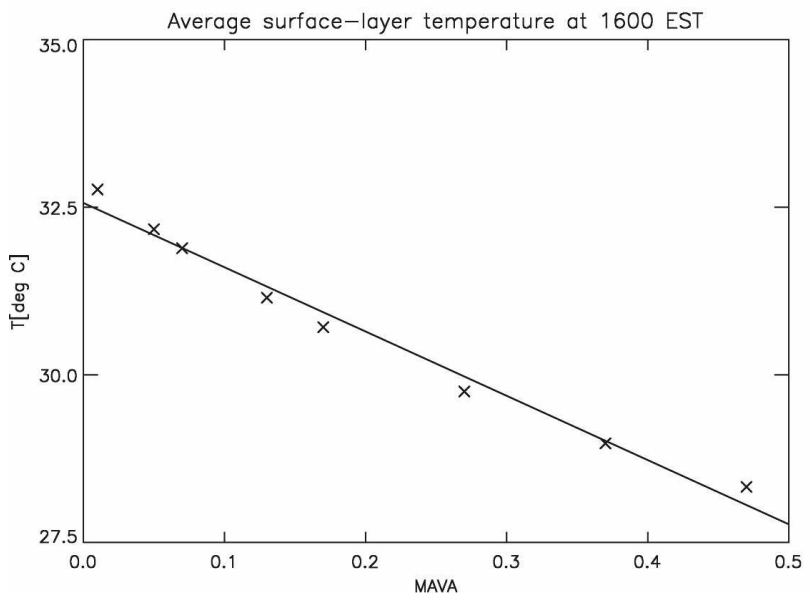

MSLP correlation coefficient. That study used the MRF scheme together with the bucket soil moisture scheme, but all other parameters were the same as those here.

Figure 5a shows the average surface temperature at 1600 EST 8 October as a function of the initial MAVA, the quantity that is used in MM5 instead of the SOILM itself. SOILM is defined as a dimensionless ratio of the volume of liquid water to that of soil. The two quantities are linked by the formula MAVA $=$ SOILM $\times 2+$ $0.09 \times(0.5-$ SOILM $)$. The average surface temperatures using the Eta and Burk-Thompson schemes would require initial values of MAVA of 0.16 and 0.24 , respectively, for the MRF scheme.

Figure $5 \mathrm{~b}$ shows values of $r_{\text {MSLP }}$ for model runs using the MRF scheme, but with different initial values for MAVA. The values given in the fourth column of Table 2 have been derived from the fit function plotted in Fig. 5b. MAVA values of 0.16 and 0.24 lead to values of $r_{\text {MSLP }}$ of 0.905 and 0.891 , respectively. Thus, the value of $r_{\text {MSLP }}$ would decrease by about $35 \%$ and $56 \%$ if the sensible heat fluxes in the surface layer were as low as those in the Eta and Burk-Thompson schemes, respectively. We conclude that the treatment of the surface layer scheme has an effect that is about as large as the inclusion of countergradient fluxes in improving the MSLP correlation in the MRF scheme.

Last, note that the use of the MSLP correlation is only one of many conceivable measures of model skill. It has an advantage here because accurate surface pressure measurements were available at all of the AWSs and surface pressure is less influenced by local

(b)

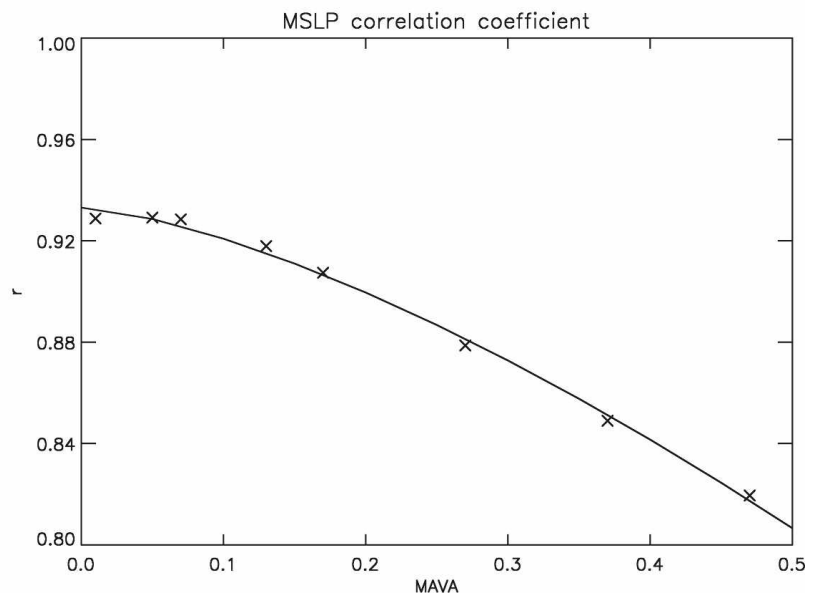

FIG. 5. (a) Average surface layer temperatures for different MAVA initializations with the MRF scheme, and (b) MSLP correlation coefficients for different MAVA initializations with the MRF scheme. The crosses indicate actual values, and the lines are [linear for (a) and quadratic for (b)] fits to these values. 
effects than, for example, surface (i.e., $10 \mathrm{~m}$ ) wind speed and direction.

\section{Conclusions}

We have shown that the skill of MM5 in predicting low-level convergence lines in the Gulf of Carpentaria region of northeastern Australia depends on the boundary layer parameterization that is chosen. We used a quantitative measure of skill based on the correlation of surface pressure between the model predictions and observations. Of the six schemes examined, the three best were those that have 1) a representation of countergradient fluxes in the convectively wellmixed layer, and 2) a treatment of the surface layer based on Monin-Obukhov theory. Of these, the MRF scheme performed marginally better than the Blackadar and Gayno-Seaman schemes. The Eta and BurkThompson schemes were less skillful and the model without a scheme was comparatively poor. The improvement in skill by including the Monin-Obukhov theory in the MRF scheme was found to be of the same magnitude as that by including the daytime countergradient fluxes. While other measures of skill could be devised, the measure based on the MSLP correlation coefficient is consistent with subjective judgments based on a comparison of the spatial patterns of lowlevel convergence with the cloud lines in satellite imagery in the case examined. The findings reported here raise important considerations for modeling the planetary boundary layer over tropical and subtropical Australia, as well as in other arid regions of the world.

\section{REFERENCES}

Berg, L. K., and S. Zhong, 2005: Sensitivity of MM5-simulated boundary layer characteristics to turbulence parameterizations. J. Appl. Meteor., 44, 1467-1483.

Betts, A. K., 1973: Non-precipitating cumulus convection and its parameterization. Quart. J. Roy. Meteor. Soc., 99, 178-196.

Billett, H., 1914: The South Wales tornado of October 27, 1913, with a note on remarkable pressure oscillations observed on Aug. 14, 1914. Geophys. Memoirs, 2, 1-17.

Blackadar, A. K., 1976: Modeling the nocturnal boundary layer. Preprints, Third Symp. on Atmospheric Turbulence, Diffusion and Air Quality, Raleigh, NC, Amer. Meteor. Soc., 4649.

- 1978: Modelling pollutant transfer during daytime convection. Preprints, Fourth Symp. on Atmospheric Turbulence, Diffusion and Air Quality, Reno, NV, Amer. Meteor. Soc., 443-447.

— 1979: High resolution models of the planetary boundary layer. Adv. Environ. Sci. Eng., 1, 50-52.

Burk, S. D., and W. T. Thompson, 1982: Operational evolution of a turbulence closure model forecast system. Mon. Wea. Rev., 110, 1535-1543.

Christie, D. R., and K. J. Muirhead, 1983: Solitary waves: A low- level wind shear hazard to aviation. Int. J. Aviat. Saf., 1, 169190.

Clarke, R. H., 1986: Several atmospheric bores and cold fronts over southern Australia. Aust. Meteor. Mag., 34, 65-76.

Deng, A., and D. R. Stauffer, 2006: On improving 4-km mesoscale model simulations. J. Appl. Meteor. Climatol., 45, 361-381.

Dudhia, J., 1989: Numerical study of convection observed during the winter monsoon experiment using a mesoscale twodimensional model. J. Atmos. Sci., 46, 3077-3107.

Egger, J., 1985: Die berliner nebelwellen. Meteor. Rundsch., 38, 103-108.

Goler, R. A., M. J. Reeder, R. K. Smith, S. Arnup, H. Richter, T. D. Keenan, P. May, and J. Hacker, 2006: Low-level convergence lines over northeastern Australia. Part I: The north Australian cloud line. Mon. Wea. Rev., 134, 3092-3108.

Grell, G. A., 1993: Prognostic evaluation of assumptions used by cumulus parameterizations. Mon. Wea. Rev., 121, 764-787.

Haase, S. P., and R. K. Smith, 1984: Morning glory wave clouds in Oklahoma: A case study. Mon. Wea. Rev., 112, 2078-2089.

Hoinka, K. P., and R. K. Smith, 1988: A dry cold front in southern Bavaria. Weather, 43, 255-260.

Hong, S.-Y., and H.-L. Pan, 1996: Nonlocal boundary layer vertical diffusion in a medium-range forecast model. Mon. Wea. Rev., 124, 2322-2339.

Jackson, G. E., R. K. Smith, and T. Spengler, 2002: The prediction of low-level mesoscale convergence lines over northeastern Australia. Aust. Meteor. Mag., 51, 13-23.

Janjić, Z. I., 1990: The step-mountain coordinate: Physical package. Mon. Wea. Rev., 118, 1429-1443.

, 1994: The step-mountain eta coordinate model: Further developments of convection, viscous sublayer, and turbulence closure schemes. Mon. Wea. Rev., 122, 927-945.

Lee, S.-M., W. Giori, M. Princevac, and H. J. S. Fernando, 2005: Implementation of a stable PBL turbulence parameterization for the mesoscale model MM5: Nocturnal flow in complex terrain. Bound.-Layer Meteor., 119, 109-134.

Louis, J. F., 1979: A parametric model of vertical eddy fluxes in the atmosphere. Bound.-Layer Meteor., 17, 187-202.

Mellor, G. L., and T. Yamada, 1974: A hierarchy of turbulence closure models for planetary boundary layers. J. Atmos. Sci., 31, 1791-1806.

Reeder, M. J., and R. K. Smith, 1998: Mesoscale meteorology. Meteorology of the Southern Hemisphere, Meteor. Monogr., No. 49, Amer. Meteor. Soc., 201-241.

Shafran, P. C., N. L. Seaman, and G. A. Gayno, 2000: Evaluation of numerical predictions of boundary layer structure during the Lake Michigan ozone study. J. Appl. Meteor., 39, 412426.

Smith, R. K., 1986: Evening glory wave-cloud lines in northwestern Australia. Aust. Meteor. Mag., 34, 27-33.

— M. J. Reeder, P. May, and H. Richter, 2006: Low-level convergence lines over northeastern Australia. Part II: Southerly disturbances. Mon. Wea. Rev., 134, 3109-3124.

Stull, R. B., 1988: An Introduction to Boundary Layer Meteorology. Kluwer Academic, 680 pp.

Therry, G., and P. Lacarrere, 1983: Improving the eddy kinetic energy model for planetary boundary layer description. Bound.-Layer Meteor., 25, 63-88.

Thomsen, G. L., 2006: Numerical simulations of low-level convergence lines over north-eastern Australia. Ph.D. thesis, Me- 
teorological Institute Munich, $151 \mathrm{pp}$. [Available online at http://edoc.ub.uni-muenchen.de/archive/00005691/01/ Thomsen_Gerald.pdf.]

, and R. K. Smith, 2006: Simulations of low-level convergence lines over northeastern Australia. Quart. J. Roy. Meteor. Soc., 132, 691-707.

Tombrou, M., A. Dandou, C. Helmis, E. Akylas, G. Angelopoulos, H. F. V. Assimakopoulos, and N. Soulakelis, 2007: Model evaluation of the atmospheric boundary layer and mixedlayer evolution. Bound.-Layer Meteor., 124, 61-79.

Troen, I. B., and L. Mahrt, 1986: A simple model of the atmo- spheric boundary layer; sensitivity to surface evaporation. Bound.-Layer Meteor., 37, 129-148.

Weinzierl, B., R. K. Smith, M. J. Reeder, and G. Jackson, 2007: MesoLAPS predictions of low-level convergence lines over northeastern Australia. Wea. Forecasting, 22, 910-927.

Zhang, D., and R. A. Anthes, 1982: A high-resolution model of the planetary boundary layer-sensitivity tests and comparisons with sesame-79 data. J. Appl. Meteor., 21, 1594-1609.

- and W.-Z. Zheng, 2004: Diurnal cycles of surface winds and temperatures as simulated by five boundary layer parameterizations. J. Appl. Meteor., 43, 157-169. 